\title{
LIPSCHITZ EQUIVALENCE OF A CLASS OF SELF-SIMILAR SETS WITH COMPLETE OVERLAPS
}

\author{
Qiuli Guo, Hao Li, Qin Wang and Lifeng Xi* \\ Zhejiang Wanli University, Institute of Mathematics \\ Ningbo, Zhejiang, 315100, P. R. China; guoqiuli@zwu.edu.cn \\ Zhejiang Wanli University, Institute of Mathematics \\ Ningbo, Zhejiang, 315100, P. R. China; kevinlee9809@yahoo.com.cn \\ Zhejiang Wanli University, School of Computer Science and Information Technology \\ Ningbo, Zhejiang, 315100, P. R. China; qinwang@126.com \\ Zhejiang Wanli University, Institute of Mathematics \\ Ningbo, Zhejiang, 315100, P. R. China; xilifengningbo@yahoo.com
}

\begin{abstract}
Fix $r \in(0,1 / 3]$. We discuss a class of self-similar sets $\left\{K_{n}\right\}_{n \geq 1}$ with complete overlaps, where $K_{n}=\left(r K_{n}\right) \cup\left(r K_{n}+r^{n}(1-r)\right) \cup\left(r K_{n}+1-r\right)$. We prove that for any $n_{1}, n_{2} \geq 1$, $K_{n_{1}}$ and $K_{n_{2}}$ are Lipschitz equivalent.
\end{abstract}

\section{Introduction}

Suppose $\left\{h_{i}: \mathbf{R}^{m} \rightarrow \mathbf{R}^{m}\right\}_{i=1}^{k}$ are contractive similitudes. We say that a compact set $\Lambda=h_{1}(\Lambda) \cup \cdots \cup h_{k}(\Lambda)$ is a self-similar set with overlaps, if there are $i \neq j$ such that $h_{i}(\Lambda) \cap h_{j}(\Lambda) \neq \varnothing$.

Self-similar sets with overlaps have very complicated structures. For example, the open set condition (OSC), which means the overlaps are little, was introduced by Moran [18] and studied by Hutchinson [10]. Schief [24], Bandt and Graf [1] showed the relation between the open set condition and the positive Hausdorff measure. Falconer [6] proved some "generic" results on Hausdorff dimension of self-similar sets without the assumption about the open set condition. One useful notion "transversality" to study self-similar sets (or measures) with overlaps can be found e.g. in Keane, Smorodinsky and Solomyak [11], Pollicott and Simon [19], Simon and Solomyak [25] and Solomyak [26]. Feng and Lau [9], Lau and Ngai [14] studied the weak separation condition. Please refer to Bandt and Hung [2], Sumi [27] for some recent work.

For self-similar set $E_{\lambda}=E_{\lambda} / 3 \cup\left(E_{\lambda} / 3+\lambda / 3\right) \cup\left(E_{\lambda} / 3+2 / 3\right)$, a conjecture of Furstenberg says that $\operatorname{dim}_{\mathrm{H}} E_{\lambda}=1$ for any $\lambda$ irrational. Świątek and Veerman [28] proved that $\operatorname{dim}_{H} E_{\lambda}>0.767$ for every $\lambda$ irrational. Kenyon [12], Rao and Wen [23] obtained that $\mathcal{H}^{1}\left(E_{\lambda}\right)>0$ if and only if $\lambda=p / q \in \mathbf{Q}$ with $p \equiv q \not \equiv 0(\bmod 3)$. The key idea of [23] is "graph-directed struture" introduced by Mauldin and Williams [17].

In particular, Rao and Wen [23] studied the self-similar sets with "complete overlaps".

doi:10.5186/aasfm.2012.3712

2010 Mathematics Subject Classification: Primary 28A80.

Key words: Fractals, self-similar set, Lipschitz equivalence, complete overlaps.

${ }^{*}$ Corresponding author.

This work is supported by National Natural Science Foundation of China (No. 11071224), Program for Excellent Talents in University of China, NSF of Ningbo (No. 2011A610176) and Post-Doctoral Science Project of Jiangsu Province (No. 1001080c). 
Definition 1. We say that a self-similar set $\Lambda=h_{1}(\Lambda) \cup \cdots \cup h_{k}(\Lambda)$ has complete overlaps, if there are words $i_{1} i_{2} \cdots i_{n_{1}} \in\{1, \cdots, k\}^{n_{1}}, j_{1} j_{2} \cdots j_{n_{2}} \in\{1, \cdots, k\}^{n_{2}}$ with $i_{1} \neq j_{1}$ such that

$$
h_{i_{1}} \circ h_{i_{2}} \circ \cdots \circ h_{i_{\left(n_{1}\right)}}(x) \equiv h_{j_{1}} \circ h_{j_{2}} \circ \cdots \circ h_{j_{\left(n_{2}\right)}}(x) .
$$

Here (1.1) implies $h_{i_{1}} \circ h_{i_{2}} \circ \cdots \circ h_{i_{\left(n_{1}\right)}}(\Lambda)=h_{j_{1}} \circ h_{j_{2}} \circ \cdots \circ h_{j_{\left(n_{2}\right)}}(\Lambda)$.

Remark 1. The cases according to "complete ovelaps" and "OSC" are quite different. For example, suppose $h_{1}, \cdots, h_{k}$ have the same ratio $\alpha$. Then (1.1) implies $n_{1}=n_{2}=n$ and $\operatorname{dim}_{\mathrm{H}} \Lambda \leq-\frac{\log \left(k^{n}-1\right)}{\log \alpha^{n}}<-\log k / \log \alpha$. However OSC implies $\operatorname{dim}_{\mathrm{H}} \Lambda=-\log k / \log \alpha$.

Remark 2. It is shown in [23] that for $\lambda \in \mathbf{Q}, \operatorname{dim}_{\mathrm{H}} E_{\lambda}<1$ if and only if $E_{\lambda}$ has complete overlaps, $E_{2 / 3^{n}}$ has complete overlaps and $\operatorname{dim}_{\mathrm{H}} E_{2 / 3^{n}}=\log _{3} \frac{3+\sqrt{5}}{2}$ for all $n \geq 1$.

The other interesting topic on self-similar sets is their Lipschitz equivalence. Here two compact subsets $X_{1}, X_{2}$ of Euclidean spaces are said to be Lipschitz equivalent, if there is a bijection $f: X_{1} \rightarrow X_{2}$ and a constant $C>0$ such that for all $x, y \in X_{1}$,

$$
C^{-1}|x-y| \leq|f(x)-f(y)| \leq C|x-y| \text {. }
$$

If compact sets $X_{1}$ and $X_{2}$ are Lipschitz equivalent, then $\operatorname{dim}_{\mathrm{H}} X_{1}=\operatorname{dim}_{\mathrm{H}} X_{2}$. However, it is worth pointing out that Cooper and Pignataro [3], Falconer and Marsh [8], David and Semmes[4] and Wen and Xi [30] showed that two self-similar sets need not be Lipschitz equivalent although they have the same Hausdorff dimension.

For self-similar sets without overlaps, Cooper and Pignataro [3], Falconer and Marsh [8], David and Semmes [4] and Xi [32] posed some algebraic conditions upon the ratios of similitudes for two given self-similar sets without overlaps to be Lipschitz equivalent. The quasi-Lipschitz equivalence, which is weaker than the Lipschitz equivalence, was studied for self-conformal sets and Ahlfors-David regular sets in Xi [31] and Wang and Xi [29], respectively. The Lipschitz embedding of fractals can be found in Llorente and Mattila [15], Mattila and Saaranen [16] and Deng and Wen et al. [5]. Please also refer to Rao, Ruan and Yang [21] and Rao, Ruan and Wang [22].

For self-similar sets with overlaps, an interesting result is on the $\{1,3,5\}-\{1,4,5\}$ Problem which was posed by David and Semmes [4]. Let $H_{1}=\left(H_{1} / 5\right) \cup\left(H_{1} / 5+\right.$ $2 / 5) \cup\left(H_{1} / 5+4 / 5\right)$ be the $\{1,3,5\}$ self-similar set, and $H_{2}=\left(H_{2} / 5\right) \cup\left(H_{2} / 5+\right.$ $3 / 5) \cup\left(H_{2} / 5+4 / 5\right)$ the $\{1,4,5\}$ self-similar set. The problem asks about the Lipschitz equivalence of $H_{1}$ and $H_{2}$. Rao, Ruan and Xi [20] proved that $H_{1}$ and $H_{2}$ are Lipschitz equivalent. Furthermore, $\mathrm{Xi}$ and Ruan [33] proved that for given $r_{1}, r_{2}, r_{3} \in(0,1)$ with $r_{1}+r_{2}+r_{3}<1$, self-similar sets $J_{1}$ and $J_{2}$ are Lipschitz equivalent if and only if $\log r_{1} / \log r_{2} \in \mathbf{Q}$, where

$$
\begin{aligned}
& J_{1}=\left(r_{1} J_{1}\right) \cup\left(r_{2} J_{1}+1-r_{2}-r_{3}\right) \cup\left(r_{3} J_{1}+1-r_{3}\right), \\
& J_{2}=\left(r_{1} J_{2}\right) \cup\left(r_{2} J_{2}+\frac{1+r_{1}-r_{2}-r_{3}}{2}\right) \cup\left(r_{3} J_{2}+1-r_{3}\right) .
\end{aligned}
$$

$\mathrm{Xi}$ and Xiong [34] generalized the result on the $\{1,3,5\}-\{1,4,5\}$ Problem to the higher dimensional spaces. Given integers $n \geq 2$ and $m \geq 1$, for $A, B \subset\{0,1, \cdots,(n-1)\}^{m}$, let $E_{A}=\cup_{a \in A} \frac{E_{A}+a}{n}$ and $E_{B}=\cup_{b \in B} \frac{E_{B}+b}{n}$ be self-similar sets in $\mathbf{R}^{m}$. Suppose that $E_{A}$ and $E_{B}$ are totally disconnected. Then it is proved in [34] that $E_{A}$ and $E_{B}$ are 
Lipschitz equivalent if and only if $A$ and $B$ have the same cardinality. Please also refer to Wen and Xi [30], Xi and Xiong [35] and Zhu et al. [36].

We notice that for the self-similar sets considered in [20], [30], [33]--[36], the open set condition holds, which means two pieces of self-similar sets touch a little. In this paper, we will consider a quite different case according to complete overlaps.

Fix $r \in(0,1 / 3]$. For any integer $n \geq 1$, let $K_{n}$ be a self-similar set satisfying

$$
K_{n}=\left(r K_{n}\right) \cup\left(r K_{n}+r^{n}(1-r)\right) \cup\left(r K_{n}+1-r\right) .
$$

The main result of the paper is stated as follows.

Theorem 1. For any $n_{1}, n_{2} \geq 1, K_{n_{1}}$ and $K_{n_{2}}$ are Lipschitz equivalent.

Remark 3. $K_{n}$ has complete overlaps. In fact, $K_{n}$ is generated by

$$
S_{1}(x)=r x, \quad S_{2}(x)=r x+r^{n}(1-r), \quad S_{3}(x)=r x+(1-r) .
$$

Let $S_{i_{1} i_{2} \cdots i_{k}}=S_{i_{1}} \circ S_{i_{2}} \circ \cdots \circ S_{i_{k}}$ and $[1]^{t}$ be the word composed of $t$ digits 1 . Then $S_{[1]^{n} 3}(x) \equiv S_{2[1]^{n}}(x) \equiv r^{n+1} x+r^{n}(1-r)$.

Remark 4. For $r=1 / 3, K_{n}=E_{2 / 3^{n}}$ and it is shown in [23] that $\left\{K_{n}\right\}_{n}$ have the same Hausdorff dimension. In fact, the technique in [23] can deal with dimension for any self-similar set $\Lambda=\cup_{i=1}^{k}\left(\Lambda / n+b_{i}\right)$ where $n \in \mathbf{N}$ and $b_{i} \in \mathbf{Q}$ for all $i$. In this paper, fix any ratio $r(\leq 1 / 3)$ rational or irrational, for the special fractals $\left\{K_{n}\right\}_{n}$, we prove that $\left\{K_{n}\right\}_{n}$ belong to the same Lipschitz equivalent class, which implies $\left\{K_{n}\right\}_{n}$ have the same dimension.

Remark 5. For $r \in(0,1 / 3]$, let $K=\left(r^{1 / 2} K\right) \cup(r K+1-r)$ be a self-similar set without overlaps. Proposition 5 in Section 6 says that $K_{n}$ and $K$ are Lipschitz equivalent for any $n$.

We organize the paper as follows. Section 2 is the preliminaries, including the counting function $L(m)$ (Lemma 1), the graph-directed sets (Lemma 2) and their corresponding criterion for Lipschitz equivalence (Lemma 3). In Section 3, for $K_{n}$ we construct the graph-directed sets with ratio $r$ and adjacency matrix $M_{n}$ defined in (3.1). In Section 4, for $K_{1}$ we construct some graph-directed sets with ratio $r$ and the same adjacency matrix $M_{n}$. Then it follows from Lemma 3 that $K_{1}$ and $K_{n}$ are Lipschitz equivalent, hence Theorem 1 is proved. In Section 5, we obtain the Perron-Frobenius eigenvector of the matrix $M_{n}$ in terms of the counting function $L(m)$ and the Fibonacci sequence. Section 6 shows that $K_{n}$ is Lipschitz equivalent to a self-similar set $K$ (in Remark 4) without overlaps.

\section{Preliminaries}

Fix $r \in(0,1 / 3]$ and integer $n \geq 1$. Let $E, F$ be the self-similar sets satisfying

$$
\begin{aligned}
& E=r E \cup(r E+r(1-r)) \cup(r E+1-r), \\
& F=r F \cup\left(r F+r^{n}(1-r)\right) \cup(r F+1-r) .
\end{aligned}
$$

Notice that $E=K_{1}$ and $F=K_{n}$.

Therefore for proving Theorem 1, we only need to prove the following proposition (in Section 4).

Proposition 1. E and $F$ are Lipschitz equivalent. 
Given any integer $m \in\left[0,2^{n}-1\right]$, let

$$
m=x_{0} x_{1} \cdots x_{n-1} \quad\left(x_{t}=0 \text { or } 1\right),
$$

be the dyadic representation of $m$, that is $m=2^{n-1} x_{0}+2^{n-2} x_{1}+\cdots+x_{n-1}$. Then we define the counting function

$$
L(m)=L\left(x_{0} x_{1} \cdots x_{n-1}\right)=\sum_{i=0}^{n-1} x_{i},
$$

i.e., the number of digits 1 in $x_{0} x_{1} \cdots x_{n-1}$. We have the following lemma.

Lemma 1. Suppose $m=x_{0} x_{1} \cdots x_{n-1} \in \mathbf{Z} \cap\left[0,2^{n}-1\right]$. Then

$$
\begin{array}{ll}
L(2 m)=L(m) \text { and } L(2 m+1)=L(m)+1 & \text { when } m<2^{n-1}, \\
L\left(2\left(m-2^{n-1}\right)\right)=L(m)-1 \text { and } L\left(2\left(m-2^{n-1}\right)+1\right)=L(m) & \text { when } m \geq 2^{n-1} .
\end{array}
$$

Proof. When $m=x_{0} \cdots x_{n-1} \in\left[0,2^{n-1}\right)$, we have $x_{0}=0$,

$$
L(2 m)=L\left(x_{1} \cdots x_{n-1} 0\right)=L\left(0 x_{1} \cdots x_{n-1}\right)=L(m),
$$

and $L(2 m+1)=L\left(x_{1} \cdots x_{n-1} 1\right)=L\left(0 x_{1} \cdots x_{n-1}\right)+1=L(m)+1$.

When $m \in x_{0} \cdots x_{n-1}=\left[2^{n-1}, 2^{n}-1\right]$, we have $x_{0}=1$,

$$
L\left(2\left(m-2^{n-1}\right)\right)=L\left(x_{1} \cdots x_{n-1} 0\right)=L\left(1 x_{1} \cdots x_{n-1}\right)-1=L(m)-1,
$$

and $L\left(2\left(m-2^{n-1}\right)+1\right)=L\left(x_{1} \cdots x_{n-1} 1\right)=L\left(1 x_{1} \cdots x_{n-1}\right)=L(m)$.

Recall the notion of "graph-directed structure" [17] as follows.

Let $(V, \mathcal{E})$ be a directed graph, where $V=\{0, \cdots,(m-1)\}$ and $\mathcal{E}$ are vertex set and edge set respectively. Denote by $\mathcal{E}_{i, j}$ the set of edges from vertex $i$ to vertex $j$. For this graph, we consider the adjacency matrix $\mathcal{A}=\left(a_{i, j}\right)_{0 \leq i, j \leq m-1}$ defined by $a_{i, j}=\# \mathcal{E}_{i, j}$. Let $\rho(\mathcal{A})$ be the spectral radius of $\mathcal{A}$. The graph is said to be transitive, if for any vertexes $i$ and $j$, there exists a directed path starting at $i$ and ending at $j$.

To simplify the structure, we assume there exists $\tau \in(0,1)$ such that every edge $e \in \mathcal{E}$ is equipped with a contracting similitude $S_{e}: \mathbf{R}^{n} \rightarrow \mathbf{R}^{n}$ with ratio $\tau$. By [17], there exists a unique family of non-empty compact sets $\Gamma_{0}, \Gamma_{1}, \cdots, \Gamma_{m-1}$ such that for any $i$,

$$
\Gamma_{i}=\bigcup_{j=0}^{m-1} \bigcup_{e \in \mathcal{E}_{i, j}} S_{e}\left(\Gamma_{j}\right) .
$$

Here $\left\{\Gamma_{0}, \Gamma_{1}, \cdots, \Gamma_{m-1}\right\}$ are called graph-directed sets on $(V, \mathcal{E})$ with ratio $\tau$. Furthermore, we say that $\left\{\Gamma_{i}\right\}_{i=0}^{m-1}$ are dust-like graph-directed sets on $(V, \mathcal{E})$, if the right hand of (2.4) is a disjoint union for each $0 \leq i \leq m-1$.

By [17], we have the following lemma.

Lemma 2. Suppose that $\left\{\Gamma_{i}\right\}_{i=0}^{m-1}$ are dust-like graph-directed sets on a transitive graph with ratio $\tau$ and adjacency matrix $\mathcal{A}$. Then for every $i$,

$$
\operatorname{dim}_{\mathrm{H}} \Gamma_{i}=-\log \rho(\mathcal{A}) / \log \tau .
$$

Theorem 2.1 of [20] yields the following lemma for Lipschitz equivalence.

Lemma 3. Suppose $\left\{\Gamma_{i}\right\}_{i=0}^{m-1}$ and $\left\{\Gamma_{i}^{\prime}\right\}_{i=0}^{m-1}$ are dust-like graph-directed sets with the same ratio and the same adjacency matrix. Then $\Gamma_{i}$ and $\Gamma_{i}^{\prime}$ are Lipschitz equivalent for any $i$. 
For example, for $\{1,3,5\}$ set $H_{1}$ and $\{1,4,5\}$ set $H_{2}$ mentioned above, we obtained

$$
\begin{array}{ll}
\Gamma_{0}=H_{1}, & \Gamma_{0}^{\prime}=H_{2}, \\
\Gamma_{1}=H_{1} \cup\left(H_{1}+2\right), & \Gamma_{1}^{\prime}=H_{2} \cup\left(H_{2}+1\right), \\
\Gamma_{2}=H_{1} \cup\left(H_{1}+2\right) \cup\left(H_{1}+4\right), & \Gamma_{2}^{\prime}=H_{2} \cup\left(H_{2}+1\right) \cup\left(H_{2}+2\right) .
\end{array}
$$

It is pointed out in [20] that $\left\{\Gamma_{i}\right\}_{i=0}^{2}$ and $\left\{\Gamma_{i}^{\prime}\right\}_{i=0}^{2}$ have the same ratio $1 / 5$ and adjacency matrix $\left(\begin{array}{lll}1 & 1 & 0 \\ 1 & 1 & 1 \\ 1 & 1 & 2\end{array}\right)$, which implies that $\Gamma_{0}\left(=H_{1}\right)$ and $\Gamma_{0}^{\prime}\left(=H_{2}\right)$ are Lipschitz equivalent.

\section{Graph-directed structure}

Given $m=x_{0} x_{1} \cdots x_{n-1} \in\left\{0,1,2, \cdots, 2^{n}-1\right\}$, set

$$
A_{m}=\bigcup_{i_{0} i_{1} \cdots i_{n-1} \in\{0,1\}^{n}}\left(F+\sum_{t=0}^{n-1} r^{t}(1-r)\left(i_{t} x_{t}\right)\right)
$$

where $F$ is defined in (2.2). In particular, $A_{0}=F$.

Let $M_{n}=\left(g_{i, j}\right)_{0 \leq i, j \leq 2^{n}-1}$ be a $2^{n} \times 2^{n}$ integer matrix, where

$$
g_{i, j}= \begin{cases}1, & \text { if } i<2^{n-1}, j=2 i \\ 1, & \text { if } i<2^{n-1}, j=2 i+1, \\ 1, & \text { if } i \geq 2^{n-1}, j=2\left(i-2^{n-1}\right), \\ 2, & \text { if } i \geq 2^{n-1}, j=2\left(i-2^{n-1}\right)+1, \\ 0, & \text { otherwise. }\end{cases}
$$

That means

$$
M_{n}=\left(\begin{array}{ccccccccc}
1 & 1 & 0 & 0 & \cdots & 0 & 0 & 0 & 0 \\
0 & 0 & 1 & 1 & \cdots & 0 & 0 & 0 & 0 \\
\vdots & \vdots & \vdots & \vdots & & \vdots & \vdots & \vdots & \vdots \\
0 & 0 & 0 & 0 & \cdots & 1 & 1 & 0 & 0 \\
0 & 0 & 0 & 0 & \cdots & 0 & 0 & 1 & 1 \\
1 & 2 & 0 & 0 & \cdots & 0 & 0 & 0 & 0 \\
0 & 0 & 1 & 2 & \cdots & 0 & 0 & 0 & 0 \\
\vdots & \vdots & \vdots & \vdots & & \vdots & \vdots & \vdots & \vdots \\
0 & 0 & 0 & 0 & \cdots & 1 & 2 & 0 & 0 \\
0 & 0 & 0 & 0 & \cdots & 0 & 0 & 1 & 2
\end{array}\right)_{2^{n} \times 2^{n}}
$$

Proposition 2. $\left\{A_{m}\right\}_{0 \leq m \leq 2^{n}-1}$ are dust-like graph-directed sets with ratio $r$ and adjacency matrix $M_{n}$, where $A_{0}=F$.

Proof. Let $m=x_{0} x_{1} \cdots x_{n-1}$ be the dyadic representation of $m$. We will discuss two cases.

Case 1. $0 \leq m<2^{n-1}$, that is $x_{0}=0$. We only need to check

$$
r A_{2 m+1} \cup\left(r A_{2 m}+1-r\right)=A_{m} \text {. }
$$


For this, we notice that

$$
A_{m}=A_{0 x_{1} \cdots x_{n-1}}=\bigcup_{i_{1} \cdots i_{n-1} \in\{0,1\}^{n-1}}\left(F+\sum_{t=1}^{n-1} r^{t}(1-r)\left(i_{t} x_{t}\right)\right)
$$

Applying equation (2.2), we have

$$
A_{m}=T_{1} \cup T_{2} \cup T_{3},
$$

where

$$
\begin{aligned}
& T_{1}=\bigcup_{i_{1} \cdots i_{n-1} \in\{0,1\}^{n-1}}\left(r F+\sum_{t=1}^{n-1} r^{t}(1-r)\left(i_{t} x_{t}\right)\right), \\
& T_{2}=\bigcup_{i_{1} \cdots i_{n-1} \in\{0,1\}^{n-1}}\left(r F+r^{n-1}(1-r)+\sum_{t=1}^{n-1} r^{t}(1-r)\left(i_{t} x_{t}\right)\right), \\
& T_{3}=\bigcup_{i_{1} \cdots i_{n-1} \in\{0,1\}^{n-1}}\left(r F+1-r+\sum_{t=1}^{n-1} r^{t}(1-r)\left(i_{t} x_{t}\right)\right) .
\end{aligned}
$$

Therefore, we have

$$
\begin{aligned}
T_{1} \cup T_{2}= & {\left[\bigcup_{i_{1} \cdots i_{n-1} \in\{0,1\}^{n-1}} r\left(F+\sum_{t=0}^{n-2} r^{t}(1-r)\left(i_{t+1} x_{t+1}\right)+r^{n-1}(1-r)(0 \cdot 1)\right)\right] } \\
& \cup\left[\bigcup_{i_{1} \cdots i_{n-1} \in\{0,1\}^{n-1}} r\left(F+\sum_{t=0}^{n-2} r^{t}(1-r)\left(i_{t+1} x_{t+1}\right)+r^{n-1}(1-r)(1 \cdot 1)\right)\right] \\
= & \bigcup_{i_{1} \cdots i_{n-1} i_{n} \in\{0,1\}^{n}} r\left(F+\sum_{t=0}^{n-2} r^{t}(1-r)\left(i_{t+1} x_{t+1}\right)+r^{n-1}(1-r)\left(i_{n} \cdot 1\right)\right) \\
= & r A_{x_{1} \cdots x_{m} 1}=r A_{2 m+1} .
\end{aligned}
$$

In the same way, we have

$$
\begin{aligned}
T_{3} & =\bigcup_{i_{1} \cdots i_{n-1} \in\{0,1\}^{n-1}}\left(r F+1-r+\sum_{t=1}^{n-1} r^{t}(1-r)\left(i_{t} x_{t}\right)\right) \\
& =r\left[\bigcup_{i_{1} \cdots i_{n-1} i_{n} \in\{0,1\}^{n}}\left(F+\sum_{t=0}^{n-2} r^{t}(1-r)\left(i_{t+1} x_{t+1}\right)+r^{n-1}(1-r)\left(i_{n} \cdot 0\right)\right)\right]+(1-r) \\
& =r A_{x_{1} \cdots x_{n-1} 0}+(1-r) \\
& =r A_{2 m}+(1-r) .
\end{aligned}
$$
union

$$
A_{m}=r A_{2 m+1} \cup\left(r A_{2 m}+1-r\right) .
$$


Case 2. $m \geq 2^{n-1}$, that is $x_{0}=1$,

$$
A_{m}=A_{1 x_{1} \cdots x_{n-1}}=\bigcup_{i_{0}=0}^{1} \bigcup_{i_{1} \cdots i_{n-1} \in\{0,1\}^{n-1}}\left(F+\sum_{t=0}^{n-1} r^{t}(1-r)\left(i_{t} x_{t}\right)\right) \text {. }
$$

Let

$$
R_{i_{0}}=\bigcup_{i_{1} \cdots i_{n-1} \in\{0,1\}^{n-1}}\left(F+(1-r)\left(i_{0}\right)+\sum_{t=1}^{n-1} r^{t}(1-r)\left(i_{t} x_{t}\right)\right) .
$$

Then

$$
A_{m}=R_{0} \cup R_{1} \text {. }
$$

Applying (2.2), we have

$$
\begin{aligned}
R_{0}= & {\left[\bigcup_{i_{1} \cdots i_{n-1} \in\{0,1\}^{n-1}}\left(r F+\sum_{t=1}^{n-1} r^{t}(1-r)\left(i_{t} x_{t}\right)\right)\right] } \\
& \cup\left[\bigcup_{i_{1} \cdots i_{n-1} \in\{0,1\}^{n-1}}\left(r F+r^{n}(1-r)+\sum_{t=1}^{n-1} r^{t}(1-r)\left(i_{t} x_{t}\right)\right)\right] \\
& \cup\left[\bigcup_{i_{1} \cdots i_{n-1} \in\{0,1\}^{n-1}}\left(r F+(1-r)+\sum_{t=1}^{n-1} r^{t}(1-r)\left(i_{t} x_{t}\right)\right)\right] \\
= & R_{0,1} \cup R_{0,2} \cup R_{0,3} .
\end{aligned}
$$

In the same way as above, we obtain

$$
R_{0,1} \cup R_{0,2}=r A_{x_{1} \cdots x_{n-1} 1}=r A_{2 m+1} .
$$

Applying (2.2), we also have

$$
\begin{aligned}
R_{1}= & {\left[\bigcup_{i_{1} \cdots i_{n-1} \in\{0,1\}^{n-1}}\left(r F+(1-r)+\sum_{t=1}^{n-1} r^{t}(1-r)\left(i_{t} x_{t}\right)\right)\right] } \\
& \cup\left[\bigcup_{i_{1} \cdots i_{n-1} \in\{0,1\}^{n-1}}\left(r F+r^{n}(1-r)+(1-r)+\sum_{t=1}^{n-1} r^{t}(1-r)\left(i_{t} x_{t}\right)\right)\right] \\
& \cup\left[\bigcup_{i_{1} \cdots i_{n-1} \in\{0,1\}^{n-1}}\left(r F+2(1-r)+\sum_{t=1}^{n-1} r^{t}(1-r)\left(i_{t} x_{t}\right)\right)\right] \\
= & R_{1,1} \cup R_{1,2} \cup R_{1,3} .
\end{aligned}
$$

Here $R_{1,1}=R_{0,3}$. In the same way,

$$
R_{1,1} \cup R_{1,2}=r A_{2 m+1}+(1-r) \text { and } R_{1,3}=r A_{2 m}+2(1-r) \text {. }
$$

Therefore

$$
\begin{aligned}
A_{m} & =R_{0,1} \cup R_{0,2} \cup R_{0,3} \cup R_{1,1} \cup R_{1,2} \cup R_{1,3} \\
& =\left(R_{0,1} \cup R_{0,2}\right) \cup\left(R_{1,1} \cup R_{1,2}\right) \cup R_{1,3} \\
& =r A_{2 m+1} \cup\left(r A_{2 m+1}+(1-r)\right) \cup\left(r A_{2 m}+2(1-r)\right) .
\end{aligned}
$$


Notice that

$$
\begin{aligned}
& r A_{2 m+1} \subset\left[0, r+\sum_{t=1}^{n} r^{t}(1-r)\right] \subset\left[0, r+\sum_{t=1}^{\infty} r^{t}(1-r)\right) \\
&=[0,2 r) \subset[0,1-r) \quad(\text { since } r \leq 1 / 3) .
\end{aligned}
$$

Similarly,

$$
\begin{aligned}
\left(r A_{2 m+1}+(1-r)\right) & \subset[1-r, 2(1-r)), \\
\left(r A_{2 m}+2(1-r)\right) & \subset[2(1-r), \infty) .
\end{aligned}
$$

Then the above three intervals are disjoint, which implies the union (3.3) is disjoint.

This proposition follows from (3.2) and (3.3).

Remark 6. For $r=1 / 3$, Rao and Wen [23] pointed out the adjacency matrix w.r.t $F$ is $M_{n}$. Our approach to prove Proposition 2, which can deal with the case of $r$ irrational, is quite different from the technique in [23].

\section{Proof of Proposition 1}

Notice that $A_{0}=F$ and $\left\{A_{m}\right\}_{0 \leq m \leq 2^{n}-1}$ is dust-like with ratio $r$ and adjacency matrix $M_{n}$. To prove the Lipschitz equivalence of $E$ and $F$, we shall construct graphdirected sets $\left\{B_{m}\right\}_{0 \leq m \leq 2^{n}-1}$ such that $B_{0}=E$ and the corresponding adjacency matrix is $M_{n}$.

Let $B_{m}$ be the set defined by

$$
B_{m}=B_{x_{0} x_{1} \cdots x_{n-1}}= \begin{cases}r^{-k} E & \text { if } L(m)=2 k, \\ r^{-k}(E \cup(E+1-r)) & \text { if } L(m)=2 k+1,\end{cases}
$$

where $E$ is defined in (2.1). Notice that $B_{0}=E$.

Lemma 4. When $m \in\left[0,2^{n-1}\right)$ and $L(m)=2 k$, we get the disjoint decomposition

$$
B_{m}=r B_{2 m+1} \cup\left(r B_{2 m}+r^{-k}(1-r)\right) .
$$

Proof. When $m=x_{0} \cdots x_{n-1} \in\left[0,2^{n-1}\right)$, we have $x_{0}=0$. Then $L(2 m)=2 k$ and $L(2 m+1)=2 k+1$ by Lemma 1 .

Applying equation (2.1), we have

$$
\begin{aligned}
B_{m} & =B_{x_{0} x_{1} \cdots x_{n-1}}=r^{-k} E \\
& =r^{-k+1} E \cup\left(r^{-k+1} E+r^{-k+1}(1-r)\right) \cup\left(r^{-k+1} E+r^{-k}(1-r)\right) \\
& =\left[r^{-k+1}(E \cup(E+1-r))\right] \cup\left[r^{-k+1} E+r^{-k}(1-r)\right] \\
& =r B_{x_{1} \cdots x_{n-1} 1} \cup\left(r B_{x_{1} \cdots x_{n-1} 0}+r^{-k}(1-r)\right) \\
& =r B_{2 m+1} \cup\left(r B_{2 m}+r^{-k}(1-r)\right) .
\end{aligned}
$$

Here $r B_{2 m+1} \subset\left[0, r^{-k+1}(2-r)\right]$ and $\left(r B_{2 m}+r^{-k}(1-r)\right) \subset\left[r^{-k}(1-r), r^{-k}\right]$, where

$$
r^{-k+1}(2-r)<r^{-k}(1-r)
$$

since $r \leq 1 / 3$. Then the decomposition in (4.2) is disjoint, which is shown in Figure 1. In this figure, we get the decomposition of $r^{-k} E$ as two parts in shadows. 


\section{$r^{-k} E$}
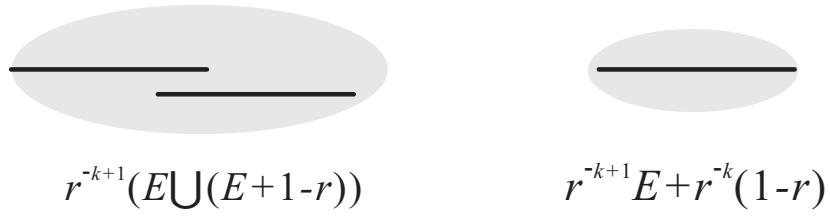

Figure 1. The decomposition of $B_{m}$ with $m \in\left[0,2^{n-1}\right)$ and $L(m)=2 k$.

Lemma 5. When $m \in\left[0,2^{n-1}\right)$ and $L(m)=2 k+1$, we get the disjoint decomposition

$$
B_{m}=r B_{2 m} \cup\left(r B_{2 m+1}+r^{-k}(1-r)\right) .
$$

Proof. When $m=x_{0} \cdots x_{n-1} \in\left[0,2^{n-1}\right)$, it should holds that $x_{0}=0$. Then $L(2 m)=2 k+1$ and $L(2 m+1)=2(k+1)$ by Lemma 1 .

Applying the equation (2.1), we have

$$
\begin{aligned}
B_{m}= & B_{x_{0} x_{1} \cdots x_{n-1}}=r^{-k} E \cup\left[r^{-k}(E+1-r)\right]=\left[r^{-k+1} E\right. \\
& \left.\cup\left(r^{-k+1} E+r^{-k+1}(1-r)\right) \cup\left(r^{-k+1} E+r^{-k}(1-r)\right)\right] \cup\left[r^{-k} E+r^{-k}(1-r)\right]
\end{aligned}
$$

We conclude that

$$
\left(r^{-k+1} E+r^{-k}(1-r)\right) \subset r^{-k} E+r^{-k}(1-r) .
$$

In fact, by (2.1), we also have

$$
\begin{aligned}
& r^{-k} E+r^{-k}(1-r) \\
& =\left(r^{-k+1} E+r^{-k}(1-r)\right) \cup\left(r^{-k+1} E+r^{-k}\left(1-r^{2}\right)\right) \cup\left(r^{-k+1} E+2 r^{-k}(1-r)\right)
\end{aligned}
$$

Therefore, we have

$$
\begin{aligned}
B_{m} & =\left[r^{-k+1} E \cup\left(r^{-k+1} E+r^{-k+1}(1-r)\right)\right] \cup\left[r^{-k} E+r^{-k}(1-r)\right] \\
& =\left[r^{-k+1}(E \cup(E+1-r))\right] \cup\left[r \cdot r^{-(k+1)} E+r^{-k}(1-r)\right] \\
& =r B_{x_{1} \cdots x_{n-1} 0} \cup\left(r B_{x_{1} \cdots x_{n-1} 1}+r^{-k}(1-r)\right) \\
& =r B_{2 m} \cup\left(r B_{2 m+1}+r^{-k}(1-r)\right) . \\
& =-r^{-k}(E \cup(E+1-r)) \\
\hline & -----
\end{aligned}
$$

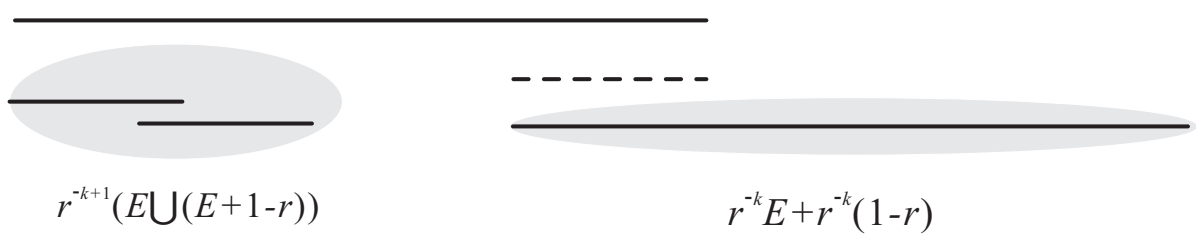

Figure 2. The decomposition of $B_{m}$ with $m \in\left[0,2^{n-1}\right)$ and $L(m)=2 k+1$. where

Here $r B_{2 m} \subset\left[0, r^{-k+1}(2-r)\right]$ and $\left(r B_{2 m+1}+r^{-k}(1-r)\right) \subset\left[r^{-k}(1-r), r^{-k}(2-r)\right]$,

$$
r^{-k+1}(2-r)<r^{-k}(1-r)
$$

since $r \leq 1 / 3$. That means the decomposition in (4.3) is disjoint as shown in Figure 2 . In this figure, we get the decomposition of $r^{-k}(E \cup(E+1-r))$ as two parts in shadows. 
Lemma 6. When $m \in\left[2^{n-1}, 2^{n}-1\right]$ and $L(m)=2 k$, we get the disjoint decomposition

$$
\begin{aligned}
B_{m}= & r B_{2\left(m-2^{n-1}\right)} \cup\left[r B_{2\left(m-2^{n-1}\right)+1}+r^{-k+1}(1-r)\right] \\
& \cup\left(r B_{2\left(m-2^{n-1}\right)+1}+r^{-k}(1-r)\right) .
\end{aligned}
$$

Proof. When $m=x_{0} x_{1} \cdots x_{n} \in\left[2^{n-1}, 2^{n}-1\right]$, it should holds that $x_{0}=1$. Then $L\left(2\left(m-2^{n-1}\right)\right)=2(k-1)+1$ and $L\left(2\left(m-2^{n-1}\right)+1\right)=2 k$ by Lemma 1 . Therefore, by $(2.1)$, we have

$$
\begin{aligned}
B_{m} & =B_{x_{0} x_{1} \cdots x_{n-1}}=r^{-k} E \\
& =r^{-k+1} E \cup\left[r^{-k+1} E+r^{-k+1}(1-r)\right] \cup\left[r^{-k+1} E+r^{-k}(1-r)\right] \\
& =Q_{1} \cup Q_{2} \cup Q_{3} .
\end{aligned}
$$

By (2.1), we also have

$$
\begin{aligned}
Q_{1} & =r^{-k+1} E \\
& =\left[r^{-k+2} E \cup\left(r^{-k+2} E+r^{-k+2}(1-r)\right) \cup\left(r^{-k+2} E+r^{-k+1}(1-r)\right)\right],
\end{aligned}
$$

where

$$
\left(r^{-k+2} E+r^{-k+1}(1-r)\right) \subset Q_{2},
$$

since

$Q_{2}=\left(r^{-k+2} E+r^{-k+1}(1-r)\right) \cup\left(r^{-k+2} E+r^{-k+1}\left(1-r^{2}\right)\right) \cup\left(r^{-k+2} E+2 r^{-k+1}(1-r)\right)$ by using (2.1) again. Hence,

$$
\begin{aligned}
B_{m} & =\left[r^{-k+2} E \cup\left(r^{-k+2} E+r^{-k+2}(1-r)\right)\right] \cup Q_{2} \cup Q_{3} \\
& =\left[r^{-k+2}(E \cup(E+1-r))\right] \cup\left(r^{-k+1} E+r^{-k+1}(1-r)\right) \cup\left(r^{-k+1} E+r^{-k}(1-r)\right) \\
& =r B_{x_{1} \cdots x_{n-1} 0} \cup\left(r B_{x_{1} \cdots x_{n-1} 1}+r^{-k+1}(1-r)\right) \cup\left(r B_{x_{1} \cdots x_{n-1} 1}+r^{-k}(1-r)\right) \\
& =r B_{2\left(m-2^{n-1}\right)} \cup\left(r B_{2\left(m-2^{n-1}\right)+1}+r^{-k+1}(1-r)\right) \cup\left(r B_{2\left(m-2^{n-1}\right)+1}+r^{-k}(1-r)\right) . \\
& r^{-k} E
\end{aligned}
$$

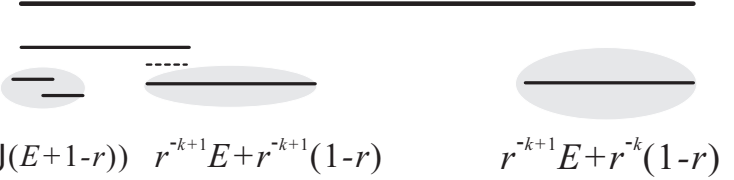

Figure 3. The decomposition of $B_{m}$ with $m \geq 2^{n-1}$ and $L(m)=2 k$.

In the same way, the decomposition in (4.4) is disjoint, which is shown in Figure 3. In this figure, we get the decomposition of $r^{-k} E$ as three parts in shadows.

Lemma 7. When $m \in\left[2^{n-1}, 2^{n}-1\right]$ and $L(m)=2 k+1$, we get the disjoint decomposition

$$
B_{m}=r B_{2\left(m-2^{n-1}\right)+1} \cup\left(r B_{2\left(m-2^{n-1}\right)+1}+r^{-k}(1-r)\right) \cup\left(r B_{2\left(m-2^{n-1}\right)}+2 r^{-k}(1-r)\right) .
$$

Proof. When $m=x_{0} \cdots x_{n-1} \in\left[2^{n-1}, 2^{n}-1\right]$, it should hold that $x_{0}=1$. Then $L\left(2\left(m-2^{n-1}\right)\right)=2 k$ and $L\left(2\left(m-2^{n-1}\right)+1\right)=2 k+1$ by Lemma 1 . By $(2.1)$, as in Figure 4, we have

$$
B_{m}=B_{x_{0} x_{1} \cdots x_{n-1}}=r^{-k} E \cup\left[r^{-k}(E+1-r)\right]=P_{1} \cup P_{2} \cup P_{3},
$$


where

$$
\begin{aligned}
& P_{1}=r^{-k+1} E \cup\left(r^{-k+1} E+r^{-k+1}(1-r)\right) \cup\left(r^{-k+1} E+r^{-k}(1-r)\right), \\
& P_{2}=\left(r^{-k+1} E+r^{-k}(1-r)\right) \cup\left(r^{-k+1} E+r^{-k+1}(1-r)+r^{-k}(1-r)\right), \\
& P_{3}=r^{-k+1} E+2 r^{-k}(1-r) . \\
& \frac{r^{-k}(E \bigcup(E+1-r))}{-} \\
& r^{-k+1}(E \bigcup(E+1-r))
\end{aligned}
$$

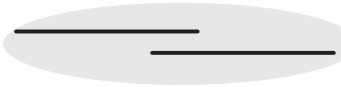

Figure 4. The decomposition of $B_{m}$ with $m \geq 2^{n-1}$ and $L(m)=2 k+1$

Notice that $P_{1}$ and $P_{2}$ have a common part $r^{-k+1} E+r^{-k}(1-r)$. Therefore, we have

$$
B_{m}=\left[r^{-k+1} E \cup\left(r^{-k+1} E+r^{-k+1}(1-r)\right)\right] \cup P_{2} \cup P_{3} .
$$

We also have

$$
r^{-k+1} E \cup\left(r^{-k+1} E+r^{-k+1}(1-r)\right)=r B_{x_{1} \cdots x_{n-1} 1},
$$

and

$$
\begin{aligned}
& P_{2}=r B_{x_{1} \cdots x_{n-1} 1}+r^{-k}(1-r), \\
& P_{3}=r B_{x_{1} \cdots x_{n-1} 0}+2 r^{-k}(1-r) .
\end{aligned}
$$

Hence

$$
B_{m}=r B_{2\left(m-2^{n-1}\right)+1} \cup\left(r B_{2\left(m-2^{n-1}\right)+1}+r^{-k}(1-r)\right) \cup\left(r B_{2\left(m-2^{n-1}\right)}+2 r^{-k}(1-r)\right) .
$$

In the same way, the decomposition in (4.5) is disjoint, which is shown in Figure 4. In this figure, we get the decomposition of $r^{-k}(E \cup(E+1-r))$ as three parts in shadows.

By Lemmas 4-7, we have the following proposition.

Proposition 3. $\left\{B_{m}\right\}_{0 \leq m \leq 2^{n}-1}$ are dust-like graph-directed sets with ratio $r$ and adjacency matrix $M_{n}$, where $B_{0}=E$.

The proof of Proposition 1. It follows from Lemma 3, Proposition 2 and Proposition 3 that $A_{0}(=F)$ and $B_{0}(=E)$ are Lipschitz equivalent. This completes the proof of Proposition 1.

\section{Properties of adjacency matrix}

In the section, for the adjacency matrix $M_{n}$ defined in (3.1), we will discuss its Perron-Frobenius eigenvalue and Perron-Frobenius eigenvector.

Let $a=\frac{1+\sqrt{5}}{2}$, we have

$$
a^{2}=a+1 \quad \text { and } \quad a^{3}=2 a+1 .
$$

Let $\left\{F_{k}\right\}_{k \geq 1}=\{1,1,2,3,5, \cdots\}$ be the Fibonacci sequence. Set $F_{-1}=1, F_{0}=0$. We have

$$
F_{k+1}=F_{k}+F_{k-1} \text { for all } k \geq 0 \text {. }
$$


By induction, we have

$$
a^{k}=a F_{k}+F_{k-1} \text { for all } k \geq 0 .
$$

In fact, $a^{0}=1=a F_{0}+F_{-1}$, assume that $a^{k}=a F_{k}+F_{k-1}$, by (5.1) and (5.2) we can find

$$
\begin{aligned}
a^{k+1} & =a\left(a F_{k}+F_{k-1}\right)=a^{2} F_{k}+a F_{k-1}=(a+1) F_{k}+a F_{k-1} \\
& =a\left(F_{k}+F_{k-1}\right)+F_{k}=a F_{k+1}+F_{k} .
\end{aligned}
$$

Proposition 4. Given $n \geq 1$, the Perron-Frobenius eigenvalue of $M_{n}$ is $\omega=$ $\frac{3+\sqrt{5}}{2}$. Let $V_{n}=\left(v_{0}, v_{1}, \cdots, v_{2^{n}-1}\right)^{T}>0$ be the Perron-Frobenius eigenvector of $M_{n}$ with $v_{0}=1$. Then

$$
V_{n}=\left(v_{m}\right)_{0 \leq m \leq 2^{n}-1}^{T} \text { with } v_{m}=a^{L(m)}=a F_{L(m)}+F_{L(m)-1} .
$$

Proof. For given $n \geq 1$, by Lemma 2 and Proposition 2, we have

$$
\operatorname{dim}_{\mathrm{H}}\left(K_{n}\right)=-\frac{\log \omega_{n}}{\log r}
$$

where $\omega_{n}$ is the Perron-Frobenius (PF) eigenvalue of $M_{n}$. In particular, we easily check that $\operatorname{dim}_{\mathrm{H}}\left(K_{1}\right)=-\frac{\log \omega_{1}}{\log r}$, where $\omega_{1}=\frac{3+\sqrt{5}}{2}$ is the PF eigenvalue of $M_{1}=$ $\left(\begin{array}{ll}1 & 1 \\ 1 & 2\end{array}\right)$

Since $K_{n}$ and $K_{1}$ are Lipschitz equivalent, we have

$$
\operatorname{dim}_{\mathrm{H}}\left(K_{n}\right)=\operatorname{dim}_{\mathrm{H}}\left(K_{1}\right) .
$$

Then by (5.5),

$$
\omega_{n}=\omega_{1}=\omega=\frac{3+\sqrt{5}}{2}\left(=a^{2}\right) .
$$

Since the matrix $M_{n}$ is primitive, we notice that the eigenspace associated to the $\mathrm{PF}$ eigenvalue is one dimensional. Therefore, to prove that $V_{n}$ defined in (5.4) is the unique PF eigenvector of $M_{n}$ with $v_{0}=a^{0}=1$, we only need to check that

$$
M_{n} V_{n}=\omega V_{n},
$$

i.e.,

$$
\sum_{j=0}^{2^{n}-1} g_{m j} v_{j}=\omega \cdot v_{m} \text { for every } m .
$$

We will check (5.6) for two cases.

Case 1. When $0 \leq m=x_{0} x_{1} \cdots x_{n-1}<2^{n-1}$, that is $x_{0}=0$. Then by Lemma 1 ,

$$
L(2 m)=L(m) \quad \text { and } \quad L(2 m+1)=L(m)+1 .
$$

By the definition of $M_{n}$ and (5.1), we have

$$
\begin{aligned}
\sum_{j=0}^{2^{n}-1} g_{m j} v_{j} & =v_{2 m}+v_{2 m+1}=a^{L(2 m)}+a^{L(2 m+1)} \\
& =a^{L(m)}+a^{L(m)+1}=a^{L(m)}(1+a)=a^{2} \cdot a^{L(m)}=\omega \cdot v_{m} .
\end{aligned}
$$

Case 2. When $2^{n-1} \leq m=x_{0} x_{1} \cdots x_{n-1} \leq 2^{n}-1$, that is $x_{0}=1$. Then by Lemma 1,

$$
L\left(2\left(m-2^{n-1}\right)\right)=L(m)-1 \quad \text { and } \quad L\left(2\left(m-2^{n-1}\right)+1\right)=L(m) .
$$


By the definition of $M_{n}$ and (5.1), we have

$$
\begin{aligned}
\sum_{j=0}^{2^{n}-1} g_{m j} v_{j} & =v_{2\left(m-2^{n-1}\right)}+2 v_{2\left(m-2^{n-1}\right)+1} \\
& =a^{L\left(2\left(m-2^{n-1}\right)\right)}+2 a^{L\left(2\left(m-2^{n-1}\right)+1\right)} \\
& =a^{L(m)-1}+2 a^{L(m)}=(1+2 a) a^{L(m)-1} \\
& =a^{3} \cdot a^{L(m)-1}=a^{2} \cdot a^{L(m)}=\omega \cdot v_{m} .
\end{aligned}
$$

Remark 7. Rao and Wen [23] pointed out that $\omega$ can be proved to be the PF eigenvalue of $M_{n}$ by induction. This conclusion is also a consequence of Proposition 1

(or Theorem 1) as shown in the proof of Proposition 4. Rao and Wen [23] also obtained the recurrent structure

$$
V_{1}=(1, a)^{T}, \cdots, V_{n}=\left(V_{n-1}, a V_{n-1}\right)^{T}, \cdots
$$

\section{Lipschitz equivalent to a self-similar set without overlaps}

Fix $r \in(0,1 / 3]$. Let

$$
K=(r K+1-r) \cup\left(r^{1 / 2} K\right)
$$

be a self-similar set without overlaps.

In this section, we will prove the following proposition.

Proposition 5. $K_{n}$ and $K$ are Lipschitz equivalent for any $n$.

Proof. By Theorem 1, we only need to show that $K_{1}$ and $K$ are Lipschitz equivalent.

In fact, let

$$
\Gamma_{0}=K \quad \text { and } \quad \Gamma_{1}=r^{-1 / 2} K
$$

Then we have the following disjoint decomposition

$$
\Gamma_{0}=K=(r K+1-r) \cup r^{1 / 2} K=\left(r \Gamma_{0}+1-r\right) \cup r \Gamma_{1} .
$$

By using (6.1), we also have the following disjoint decomposition

$$
\begin{aligned}
\Gamma_{1} & =r^{-1 / 2} K=r^{-1 / 2}\left[(r K+1-r) \cup r^{1 / 2} K\right] \\
& =\left[r\left(r^{-1 / 2} K\right)+r^{-1 / 2}(1-r)\right] \cup K \\
& =\left[r \Gamma_{1}+r^{-1 / 2}(1-r)\right] \cup\left(r \Gamma_{0}+1-r\right) \cup r \Gamma_{1} .
\end{aligned}
$$

The above decompositions (6.1)-(6.2) show that $\left\{\Gamma_{0}, \Gamma_{1}\right\}$ are dust-like graph-directed sets with ratio $r$ and adjacency matrix

$$
M_{1}=\left(\begin{array}{cc}
1 & 1 \\
1 & 2
\end{array}\right)
$$

Applying Proposition 2 to the case of $n=1$, we get graph-directed sets $\left\{B_{0}, B_{1}\right\}$ with ratio $r$ and adjacency matrix $M_{1}$, where $B_{0}=K_{1}$.

It follows from Lemma 3 that $K$ and $K_{1}$ are Lipschitz equivalent.

Acknowledgements. The corresponding author acknowledges helpful discussion with Professor Z. Y. Wen. 


\section{References}

[1] BAndt, C., and S. GRAF: Self-similar sets. VII. A characterization of self-similar fractals with positive Hausdorff measure. - Proc. Amer. Math. Soc. 114:4, 1992, 995-1001.

[2] Bandt, C., and N. V. Hung: Self-similar sets with an open set condition and great variety of overlaps. - Proc. Amer. Math. Soc. 136:11, 2008, 3895-3903.

[3] Cooper, D., and T. Pignataro: On the shape of Cantor sets. - J. Differential Geom. 28:2, 1988, 203-221.

[4] David, G., and S. SEmmes: Fractured fractals and broken dreams, self-similar geometry through metric and measure. - Oxford Univ. Press, New York, 1997.

[5] Deng, J., Z. Y. Wen, X. XIOng, and L. F. XI: Bilipschitz embedding of self-similar sets. J. Anal. Math. (to appear).

[6] Falconer, K. J.: The Hausdorff dimension of some fractals and attractors of overlapping construction. - J. Statist. Phys. 47:1-2, 1987, 123-132.

[7] Falconer, K. J., and D. T. Marsh: Classification of quasi-circles by Hausdorff dimension. Nonlinearity 2:3, 1989, 489-493.

[8] Falconer, K. J., and D. T. Marsh: On the Lipschitz equivalence of Cantor sets. - Mathematika 39:2, 1992, 223-233.

[9] FenG, D. J., and K. S. LAU: Multifractal formalism for self-similar measures with weak separation condition. - J. Math. Pures Appl. 92:4, 2009, 407-428.

[10] Hutchinson, J. E.: Fractals and self-similarity. - Indiana Univ. Math. J. 30:5, 1981, $713-747$.

[11] Keane, M., M. Smorodinsky, and B. Solomyak: On the morphology of $\gamma$-expansions with deleted digits. - Trans. Amer. Math. Soc. 347:3, 1995, 955-966.

[12] Kenyon, R.: Projecting the one-dimensional Sierpinski gasket. - Israel J. Math. 97, 1997, $221-238$.

[13] Kenyon, R., and Y. Peres: Intersecting random translates of invariant Cantor sets. - Invent. Math. 104:3, 1991, 601-629.

[14] LAU, K.S., and S. M. NGAI: Multifractal measures and a weak separation condition. - Adv. Math. 141:1, 1999, 45-96.

[15] Llorente, M., and P. Mattila: Lipschitz equivalence of subsets of self-conformal sets. Nonlinearity 23:4, 2010, 875-882.

[16] Mattila, P., and P. SaAranen: Ahlfors-David regular sets and bilipschitz maps. - Ann. Acad. Sci. Fenn. Math. 34:2, 2009, 487-502.

[17] Mauldin, R. D., and S. C. Williams: Hausdorff dimension in graph directed constructions. - Trans. Amer. Math. Soc. 309:2, 1988, 811-829.

[18] Moran, P. A. P.: Additive functions of intervals and Hausdorff measure. - Proc. Cambridge Philos. Soc. 42, 1946, 15-23.

[19] Pollicott, M., and K. Simon: The Hausdorff dimension of $\gamma$-expansions with deleted digits. - Trans. Amer. Math. Soc. 347:3, 1995 967-983.

[20] RaO, H., H. J. RuAn, and L. F. XI: Lipschitz equivalence of self-similar sets. - C. R. Math. Acad. Sci. Paris 342:3, 2006, 191-196.

[21] RAO, H., H. J. RuAn, and Y. M. YANG: Gap sequence, Lipschitz equivalence and box dimension of fractal sets. - Nonlinearity 21:6, 2008, 1339-1347.

[22] RAO, H., H. J. RuAn, and Y. WANG: Lipschitz equivlance of Cantor sets and algebraic properties of contraction ratios. - Trans. Amer. Math. Soc. (to appear).

[23] RAO, H., and Z. Y. WEN: A class of self-similar fractals with overlap structure. - Adv. in Appl. Math. 20:1, 1998, 50-72. 
[24] SCHIEF, A.: Separation properties for self-similar sets. - Proc. Amer. Math. Soc. 122:1, 1994, $111-115$.

[25] Simon, K., and B. Solomyak: On the dimension of self-similar sets. - Fractals 10:1, 2002, $59-65$.

[26] Solomyak, B.: On the random series $\sum \pm \lambda^{n}$ (an Erdös problem). - Ann. of Math. (2) 142:3, 1995, 611-625.

[27] Sumi, H.: Interaction cohomology of forward or backward self-similar systems. - Adv. Math. 222:3, 2009, 729-781.

[28] Świştek, G., and J. J. P. Veerman: On a conjecture of Furstenberg. - Israel J. Math. 130, $2002,145-155$.

[29] Wang, Q., and L. F. XI: Quasi-Lipschitz equivalence of Ahlfors-David regular sets. - Nonlinearity 24, 2011, 941-950.

[30] Wen, Z. Y., and L. F. XI: Relations among Whitney sets, self-similar arcs and quasi-arcs. Israel J. Math. 136, 2003, 251-267.

[31] XI, L. F.: Quasi-Lipschitz equivalence of fractals. - Israel J. Math. 160, 2007, 1-21.

[32] XI, L. F.: Lipschitz equivalence of dust-like self-similar sets. - Math. Z. 266:3, 2010, 683-691.

[33] XI, L. F., and H. J. RuAN: Lipschitz equivalence of generalized $\{1,3,5\}-\{1,4,5\}$ self-similar sets. - Sci. China Ser. A 50:11, 2007, 1537-1551.

[34] XI, L. F., and Y. XIOng: Self-similar sets with initial cubic patterns. - C. R. Math. Acad. Sci. Paris 348:1-2, 2010, 15-20.

[35] XI, L. F., and Y. XIONG: Lipschitz equivalence of fractals generated by nested cubes. - Math. Z. (to appear), doi:10.1007/s00209-011-0916-5.

[36] ZHU, Z. Y., Y. XIONG, and L. F. XI: Lipschitz equivalence of self-similar sets with triangular pattern. - Sci. China Ser. A 54:5, 2011, 1019-1026.

Received 20 May 2011 